\title{
Direct measurements and detection techniques with low-energy RIBs
}

\author{
H. Yamaguchi ${ }^{1, \star}$, S. Hayakawa ${ }^{1}$, L. Yang ${ }^{1}$, H. Shimizu ${ }^{1}$, D. Kahl ${ }^{2,1}$, and CRIB collaboration \\ ${ }^{1}$ Center for Nuclear Study (CNS), University of Tokyo, RIKEN campus, 2-1 Hirosawa, Wako, Saitama 351- \\ 0198, Japan \\ ${ }^{2}$ School of Physics and Astronomy, the University of Edinburgh, Peter Guthrie Tait Road, Edinburgh EH9 \\ 3BF, UK
}

\begin{abstract}
Astrophysical reactions involving radioactive isotopes (RI) are of importance for the stellar energy generation and nucleosynthesis especially in high-temperature astrophysical sites, such as X-ray bursts, core-collapse supernovae, and supermassive metalpoor stars. In spite of the essential difficulties in the experimental evaluation of those reaction rates, there are several successful approaches to study them, owing to the recent technical developments in the beam production, measurement method, and detectors. Among them, the measurements of $\alpha$ resonant scattering and $(\alpha, p)$ reactions using the thick-target method in inverse kinematics are discussed. The experiments at the low-energy RI beam separator CRIB, operated by Center for Nuclear Study (CNS), the University of Tokyo, are introduced as examples for such studies.
\end{abstract}

\section{Introduction}

The essential difficulty in experimental nuclear astrophysics lies in reproducing the special conditions of stellar environments. In stars, vast amount of atoms can be condensed with an extremely high density and nuclear reactions with tiniest cross sections can be effective. Some processes are slow and may take more than millions of years, which is much longer than our life, but stars can live such long to complete the process. The typical temperature of stellar burning sites is $10^{7}-10^{9} \mathrm{~K}$, which can be regarded as a high temperature, but lower than the temperature corresponding to the Coulomb barrier of the nucleus. This implies the astrophysical reactions may occur slowly, making the experiment to simulate them extremely difficult.

Some astrophysical reactions involve radioactive isotopes (RI). Although the RI are seldom seen on the earth, they may play an important role in stars. An example is the CNO cycle, which is a basic energy generation process in stars, taking place even in our sun. In the CNO cycle, the radioactive ${ }^{13} \mathrm{~N}$ nucleus is produced via the ${ }^{12} \mathrm{C}(p, \gamma){ }^{13} \mathrm{~N}$ reaction. The $\beta$-decay half life of ${ }^{13} \mathrm{~N}$ is 10 minutes, which may restrict the speed of the cycle. If the cycle includes ${ }^{14} \mathrm{O}$ by the ${ }^{13} \mathrm{~N}(p, \gamma){ }^{14} \mathrm{O}$ reaction (the hot-CNO cycle), the slow $\beta$-decay of ${ }^{13} \mathrm{~N}$ is bypassed, and the cycle is accelerated, resulting in a higher energy generation rate.

^e-mail: yamag@cns.s.u-tokyo.ac.jp 
The earliest study of astrophysical reaction with RI beam was in fact on this ${ }^{13} \mathrm{~N}(p, \gamma){ }^{14} \mathrm{O}$ reaction $[1,2]$. Such measurements with RI beam often suffer from the limitation of beam intensities. The intensity of RI beam is typically $10^{5}$ particles per second (pps), while $>10^{14}$ pps is available for light-ion beams. This great difference in the beam intensity is fundamental for the feasibility of the measurement. Another limitation is the target. In a normal experimental condition, short-lived RI or neutrons can not be used as the target, and can only be used as the beam.

As far as we discuss astrophysical implication of RI-involving reactions, there are two favorable facts which should be noted: 1) In most cases the RI in stars play an effective role in high-temperature (explosive) phenomena, where the reaction cross sections are not too small. 2) We may think about some reaction involving very exotic nuclei and having a very small cross section, which is extremely difficult to be measured. In such a case, however, the reaction should be of small or no importance for the astrophysics.

In spite of the technical limitation, there are several successful experimental approaches:

- Direct measurement for studying high-temperature phenomena

This is feasible with relatively high-intensity RI beam $\left({ }^{7} \mathrm{Be},{ }^{13} \mathrm{~N}[2]\right.$ etc.), or high-cross section reactions (such as $(\alpha, p)$ reactions).

- Studying resonance properties

Some reactions proceed predominantly via resonant reactions, and we only need to study the property of resonances by transfer reactions [3], resonant scatterings [4], or some other methods.

- Indirect measurement with RI beams

Indirect measurements with the Asymptotic Normalization Coefficient (ANC) [5, 6], Coulomb breakup [1,7] or Trojan horse method [8] can be carried out even with RI beams.

In this article, we particularly discuss on the measurement of ${ }^{7} \mathrm{Be}+\alpha$ resonant scattering and the direct measurement of ${ }^{11} \mathrm{C}(\alpha, p)$ reaction with the thick-target method in inverse kinematics (TTIK) as successful examples.

\section{Thick-target method in inverse kinematics}

The TTIK [9] is a method suitable for astrophysical reaction studies. In that method, the beam energy is degraded in a thick reaction target, and the reaction occurs at various center-of-mass energies. By detecting the position and the energy of light particles emitted after the reaction, the reaction position in the target and the reaction energy are deduced according to the kinematical relationship. This method has several advantages, namely, (a) using inverse kinematics, we can study reactions with short-lived RI which cannot be used as the target, (b) we can perform a simultaneous measurement of cross section for a certain energy range without varying the incoming RI beam energy, (c) when the beam is stopped in the target, we can perform measurements at $180^{\circ}$ in center-of-mass angle, where the Coulomb scattering is minimal, and (d) the inverse kinematics condition can be helpful for a high resolution measurement at the energy region lower than the beam energy.

Many proton- and $\alpha$-resonant scattering [4, 10-13] experiments have been performed with the TTIK method at CRIB $[14,15]$, which is an RI beam separator operated by Center for Nuclear Study (CNS), the University of Tokyo. Most of those studies are related to the astrophysical $(p, \gamma)$ or $(\alpha, \gamma)$ capture reactions. Direct measurement of $(\alpha, p)$ reactions, such as ${ }^{14} \mathrm{O}(\alpha, p)$ [16], ${ }^{11} \mathrm{C}(\alpha, p)$ [17], ${ }^{21} \mathrm{Na}(\alpha, p),{ }^{18} \mathrm{Ne}(\alpha, p)$, and ${ }^{22} \mathrm{Mg}(\alpha, p)$, have also been performed with the TTIK method using RI beams at CRIB. 


\section{$2.1{ }^{7} \mathrm{Be}+\alpha$ elastic resonant scattering}

A typical example of the resonant scattering experiment at CRIB is for the ${ }^{7} \mathrm{Be}+\alpha$ system. The measurement allows us to evaluate the rate of the ${ }^{7} \mathrm{Be}(\alpha, \gamma)$ reaction, which is considered to play an important role in the hot $p$ - $p$ chain and related reaction sequences [18]. Several reaction sequences including the ${ }^{7} \mathrm{Be}(\alpha, \gamma)$ reaction should take place in some high-temperature environments at $T_{9}>0.2$, where $T_{9}$ is the temperature in GK. During the $v p$-process in core-collapse supernovae [19], the ${ }^{7} \mathrm{Be}(\alpha, \gamma)$ reaction may contribute as much as the triple- $\alpha$ process to the synthesis of elements heavier than boron at the relevant temperature of $T_{9}=1.5-3$ [20]. The Gamow energy window at the highest temperature $T_{9}=3$ corresponds to the excitation energy $E_{\mathrm{ex}}=8.2-9.6 \mathrm{MeV}$ in ${ }^{11} \mathrm{C}$.

In the measurement [4], a low-energy ${ }^{7} \mathrm{Be}$ beam at $14.7 \mathrm{MeV}$ was produced using a $2.3-\mathrm{mg} / \mathrm{cm}^{2}$ thick hydrogen gas target and a primary ${ }^{7} \mathrm{Li}$ beam at $5.0 \mathrm{MeV} / \mathrm{u}$. The typical ${ }^{7} \mathrm{Be}$ beam intensity was $1-2 \times 10^{5}$ per second at the secondary target, and the main measurement using a thick heliumgas target was performed for 4 days, injecting $2.9 \times 10^{10}{ }^{7} \mathrm{Be}$ particles into the target. We obtained an excitation function of the elastic scattering with several peaks corresponding to the resonance structure in ${ }^{11} \mathrm{C}$. The obtained excitation function is shown in the left panel of Figure 1. An R-matrix analysis was performed to deduce the parameters of the resonances, as the calculated curve also shown in the figure. A similar measurement was independently carried out by M. Freer et al. at other facilities [21], but our measurement included $\gamma$-ray detection to identify inelastic scattering events, and several differences were found in the obtained spectra [4], such as a shift in resonance energy by $500 \mathrm{keV}$ and almost factor 2 difference in the absolute cross section.

The observed resonances may contribute to the astrophysical ${ }^{7} \mathrm{Be}(\alpha, \gamma){ }^{11} \mathrm{C}$ reaction rate at high temperature, $T_{9}>1.5$. We calculated the resonant reaction rate and compared it with the evaluation in NACRE [22, 23]. In the NACRE evaluation, only 2 resonances at 8.1045 and $8.420 \mathrm{MeV}$ are included. These two resonances dominate the reaction rate $N_{\mathrm{A}}\langle\sigma v\rangle$ up to the temperature $T_{9} \sim 3$, and a Hauser-Feshbach calculation was used to provide the reaction rate at higher temperatures. The resonant reaction rates were calculated for four resonance parameter sets using analytical formula described in [22], as in Fig. 1. In conclusion, the resonances at $8.90 \mathrm{MeV}$ and $9.20 \mathrm{MeV}$ have a possibility to give significant contributions to the reaction rate for $T_{9}=1.5-3$, although they are unlikely to be comparable to the dominant contribution of the $8.420-\mathrm{MeV}$ resonance.

\subsection{Direct measurement of the ${ }^{11} \mathbf{C}(\alpha, p)$ reaction}

$(\alpha, p)$ reactions often play an important role in the burning process [24] taking place at high temperature stellar sites, such as, X-ray bursts, core-collapse supernovae, and supermassive metal-poor stars. ${ }^{11} \mathrm{C}(\alpha, p)$ is one of the key reactions in the $v p$-process [20], and the first direct measurement was performed at CRIB [17] with the TTIK. The experimental setup was essentially similar to the $\alpha$ resonant scattering, as shown in Figure 2.

The TTIK is an efficient method as it allows us to measure reaction events occurring at various energies simultaneously. However, it implies the identification of the exit channel may not be complete, because the particles with different origins (such as elastic scattering and inelastic scattering) can be detected at the same detector position with the same energy. This is not a crucial problem for the resonant elastic scattering, in which the cross section is much enhanced by the interference between the nuclear and Coulomb scatterings. For the $(\alpha, p)$ reactions, however, the reaction with the transition to the excited states in the residual nucleus should be accounted for.

With the setup as shown in Figure 2 we identified the reactions with the transition to different states in the excited ${ }^{14} \mathrm{O}$, namely, ${ }^{11} \mathrm{C}\left(\alpha, p_{0}\right),{ }^{11} \mathrm{C}\left(\alpha, p_{1}\right)$, and ${ }^{11} \mathrm{C}\left(\alpha, p_{2}\right)$ with the time-of-flight information. We evaluated the contribution of each of these reactions separately, and concluded that the $\left(\alpha, p_{1}\right)$ and 

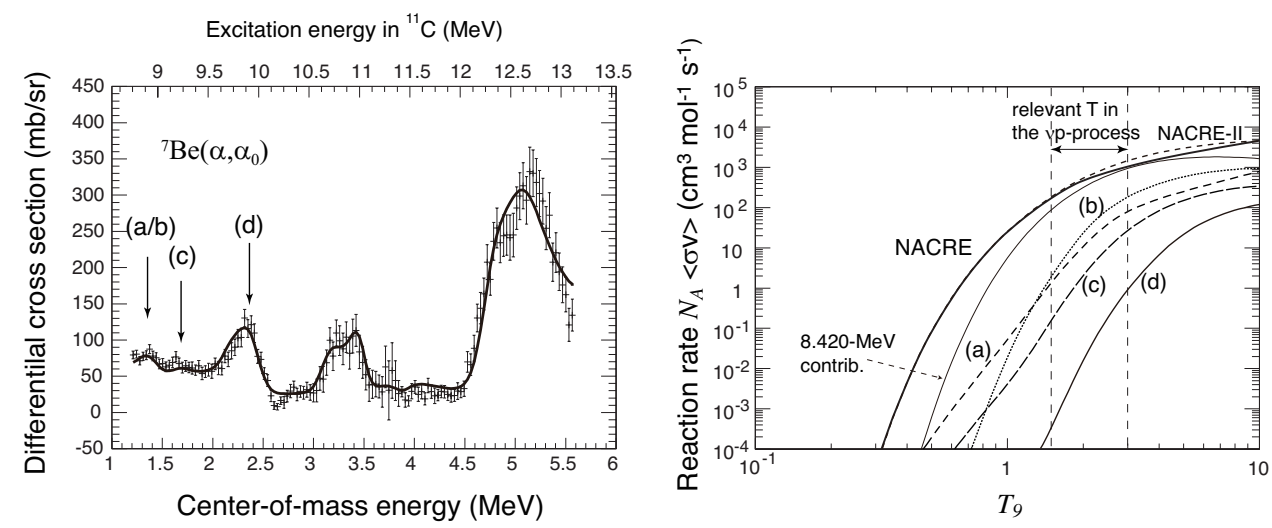

Figure 1. Excitation function of ${ }^{7} \mathrm{Be}+\alpha$ elastic scattering with an R-matrix fit curve (left panel) and evaluated resonant reaction rates of the ${ }^{7} \mathrm{Be}(\alpha, \gamma)$ reaction for the $8.90,9.20$, and $9.97-\mathrm{MeV}$ resonances, calculated by the analytical formula. (a) and (b) are for the same resonance, but with different spin-parity. The evaluation by NACRE [22] and NACRE-II [23] are shown for comparison. The contribution by the $8.420-\mathrm{MeV}$ resonance, included in NACRE, is also shown.

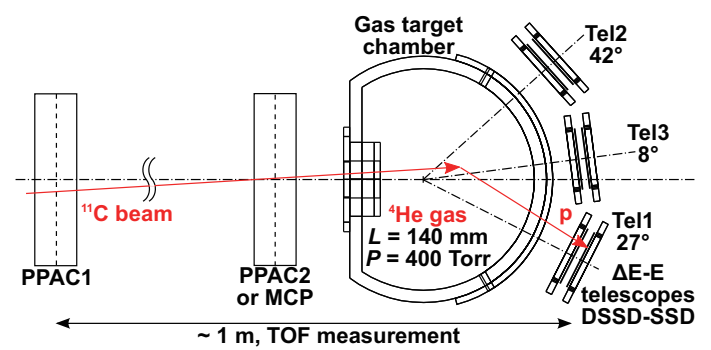

Figure 2. Experimental setup for the ${ }^{11} \mathrm{C}(\alpha, p)$ reaction measurement [17]

$\left(\alpha, p_{2}\right)$ reactions may enhance the reaction rate by $20 \%$ at most (see [17] for details). The reaction had been studied also by the reverse ${ }^{14} \mathrm{~N}\left(p_{0}, \alpha\right)$ reaction [25], however, such contribution could only be evaluated by the ${ }^{11} \mathrm{C}(\alpha, p)$ reaction measurement.

\section{Active target for an advanced form of TTIK}

We have developed an active target, referred to as "GEM-MSTPC" [26], for an improved TTIK measurement. That active target is basically a time projection chamber (TPC) with gas electron multiplier (GEM) readout, operated with helium $(90 \%)+\mathrm{CO}_{2}(10 \%)$ gases in the chamber. The helium gas serves as the reaction target and the detector gas simultaneously, and we can detect the 3-dimensional trajectories of the particles directly in the gas target. For the TTIK method, this active target provides additional information compared to the static-target experiment; the trajectory and energy loss for each of the beam particle and the reaction products. This covers the disadvantage of the normal thick target method, the potential misidentification of reactions when there are two or more possible reactions. 
Several measurements have been performed with this active target, including the ${ }^{30} \mathrm{~S}+\alpha$ elastic scattering [13]. In the ${ }^{30} \mathrm{~S}+\alpha$ experiment, we successfully obtained the Bragg curve of the beam particle in the target, and the elastic scattering events were clearly identified. However, the advantage of the active target was preliminary in that experiment, because of the limited energy and position resolution of TPC. Nevertheless, new resonances are observed and we evaluated the resonant contribution to the ${ }^{30} \mathrm{~S}(\alpha, p)$ reaction, which is one of the most effective reaction for the energy generation in X-ray bursts [27].

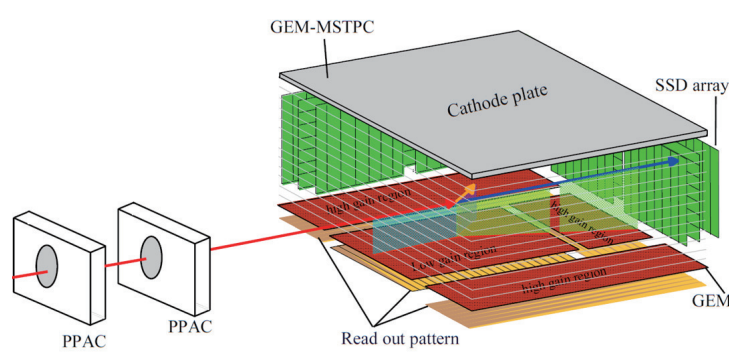

Figure 3. Active target "GEM-MSTPC" of CRIB

\section{Summary}

In spite of the technical difficulties in studying astrophysical reactions involving RI, there are several approaches for the determination of reaction rates directly or indirectly. The TTIK is one striking method to study astrophysical reactions with RI beams. So far, proton or $\alpha$ resonant scattering experiments to study properties of resonances, and direct measurement of $(\alpha, p)$ reactions are successfully applied for the experimental evaluation of astrophysical reaction rates. More advanced techniques for the RI beam production, measurement scheme and detection system would be required for a systematic understanding of RI-involving astrophysical reactions. The active target is one such technique which can cover the present disadvantages of the TTIK.

\section{Acknowledgments}

The experiments were performed at RI Beam Factory operated by RIKEN Nishina Center and CNS, the University of Tokyo. We are grateful to the RIKEN and CNS accelerator staff for their help. This work was partly supported by JSPS KAKENHI (Nos. 25800125, 15K17662, 16K05369, and 16H03980) from the Ministry of Education, Culture, Sports, Science and Technology (MEXT) of Japan.

\section{References}

[1] T. Motobayashi, T. Takei, S. Kox, C. Perrin, F. Merchez, D. Rebreyend, K. Ieki, H. Murakami, Y. Ando, N. Iwasa et al., Physics Letters B 264, 259 (1991)

[2] P. Decrock, T. Delbar, P. Duhamel, W. Galster, M. Huyse, P. Leleux, I. Licot, E. Liénard, P. Lipnik, M. Loiselet et al., Phys. Rev. Lett. 67, 808 (1991) 
[3] D.W. Bardayan, J.A. Howard, J.C. Blackmon, C.R. Brune, K.Y. Chae, W.R. Hix, M.S. Johnson, K.L. Jones, R.L. Kozub, J.F. Liang et al., Phys. Rev. C 74, 045804 (2006)

[4] H. Yamaguchi, D. Kahl, Y. Wakabayashi, S. Kubono, T. Hashimoto, S. Hayakawa, T. Kawabata, N. Iwasa, T. Teranishi, Y. Kwon et al., Phys. Rev. C 87, 034303 (2013)

[5] Z.H. Li, B. Guo, S.Q. Yan, G. Lian, X.X. Bai, Y.B. Wang, S. Zeng, J. Su, B.X. Wang, W.P. Liu et al., Phys. Rev. C 74, 035801 (2006)

[6] O.R. Tojiboev, R. Yarmukhamedov, S.V. Artemov, S.B. Sakuta, Phys. Rev. C 94, 054616 (2016)

[7] F. Schümann, S. Typel, F. Hammache, K. Sümmerer, F. Uhlig, I. Böttcher, D. Cortina, A. Förster, M. Gai, H. Geissel et al., Phys. Rev. C 73, 015806 (2006)

[8] S. Cherubini, M. Gulino, C. Spitaleri, G.G. Rapisarda, M. La Cognata, L. Lamia, R.G. Pizzone, S. Romano, S. Kubono, H. Yamaguchi et al., Phys. Rev. C 92, 015805 (2015)

[9] K.P. Artemov, O.P. Belyanin, A.L. Vetoshkin, R. Wolskj, M.S. Golovkov, V.Z. Gol'dberg, M. Madeja, V.V. Pankratov, I.N. Serikov, V.A. Timofeev et al., Sov. J. Nucl. Phys 52, 408 (1990)

[10] H. Yamaguchi, Y. Wakabayashi, S. Kubono, G. Amadio, H. Fujikawa, T. Teranishi, A. Saito, J. He, S. Nishimura, Y. Togano et al., Phys. Lett. B 672, 230 (2009)

[11] J.J. He, L.Y. Zhang, A. Parikh, S.W. Xu, H. Yamaguchi, D. Kahl, S. Kubono, J. Hu, P. Ma, S.Z. Chen et al., Phys. Rev. C 88, 012801 (2013)

[12] H. Yamaguchi, T. Hashimoto, S. Hayakawa, D.N. Binh, D. Kahl, S. Kubono, Y. Wakabayashi, T. Kawabata, T. Teranishi, Phys. Rev. C 83, 034306 (2011)

[13] D. Kahl, H. Yamaguchi, S. Kubono, A.A. Chen, A. Parikh, D.N. Binh, J. Chen, S. Cherubini, N.N. Duy, T. Hashimoto et al., Phys. Rev. C 97, 015802 (2018)

[14] S. Kubono, Y. Yanagisawa, T. Teranishi, S. Kato, T. Kishida, S. Michimasa, Y. Ohshiro, S. Shimoura, K. Ue, S. Watanabe et al., Eur. Phys. J. A13, 217 (2002)

[15] Y. Yanagisawa, S. Kubono, T. Teranishi, K. Ue, S. Michimasa, M. Notani, J.J. He, Y. Ohshiro, S. Shimoura, S. Watanabe et al., Nucl. Instrum. Meth. Phys. Res., Sect. A 539, 74 (2005)

[16] A. Kim, N.H. Lee, M.H. Han, J.S. Yoo, K.I. Hahn, H. Yamaguchi, D.N. Binh, T. Hashimoto, S. Hayakawa, D. Kahl et al., Phys. Rev. C 92, 035801 (2015)

[17] S. Hayakawa, S. Kubono, D. Kahl, H. Yamaguchi, D.N. Binh, T. Hashimoto, Y. Wakabayashi, J.J. He, N. Iwasa, S. Kato et al., Phys. Rev. C 93, 065802 (2016)

[18] M. Wiescher, J. Görres, S. Graff, L. Buchmann, F.K. Thielemann, Astrophys. J. 343, 352 (1989)

[19] C. Fröhlich, P. Hauser, M. Liebendörfer, G. Martínez-Pinedo, F.K. Thielemann, E. Bravo, N. Zinner, W. Hix, K. Langanke, A. Mezzacappa et al., Astrophys. J. 637, 415 (2006)

[20] S. Wanajo, H.T. Janka, S. Kubono, Astrophys. J. 729, 46 (2011)

[21] M. Freer, N.L. Achouri, C. Angulo, N.I. Ashwood, D.W. Bardayan, S. Brown, W.N. Catford, K.A. Chipps, N. Curtis, P. Demaret et al., Phys. Rev. C 85, 014304 (2012)

[22] C. Angulo et al., Nucl. Phys. A 656, 3 (1999)

[23] Y. Xu, K. Takahashi, S. Goriely, M. Arnould, M. Ohta, H. Utsunomiya, Nucl. Phys. A 918, 61 (2013)

[24] R.K. Wallace, S.E. Woosley, Astrophys. J. Suppl. Ser. 45, 389 (1981)

[25] P.D. Ingalls, J.S. Schweitzer, B.D. Anderson, M. Rios, Phys. Rev. C 13, 524 (1976)

[26] T. Hashimoto, H. Ishiyama, T. Ishikawa, T. Kawamura, K. Nakai, Y. Watanabe, H. Miyatake, M. Tanaka, Y. Fuchi, N. Yoshikawa et al., Nuclear Instruments and Methods in Physics Research Section A 556, 339 (2006)

[27] R. Cyburt, A. Amthor, A. Heger, E. Johnson, L. Keek, Z. Meisel, H. Schatz, K. Smith, The Astrophysical Journal 830, 55 (2016) 